\title{
Numerical Investigation of Temperature Distribution in a Homogeneous Aquifer Thermal Energy Storage System During Thermal Injection Process
}

\author{
Mohammed Hirpho Tobe ${ }^{1}$, Zerihun Kinfe Birhanu ${ }^{2}$ \\ ${ }^{1}$ Department of Mathematics, Ambo University, Ambo, Ethiopia \\ ${ }^{2}$ Department of Mathematics, Hawassa University, Hawassa, Ethiopia \\ Email address: \\ mohhirpho@gmail.com (M. H. Tobe), zerie96@gmail.com (Z. K. Birhanu) \\ To cite this article: \\ Mohammed Hirpho Tobe, Zerihun Kinfe Birhanu. Numerical Investigation of Temperature Distribution in a Homogeneous Aquifer Thermal \\ Energy Storage System During Thermal Injection Process. American Journal of Applied Mathematics. Vol. 8, No. 3, 2020 , pp. 89-97. \\ doi: $10.11648 /$ j.ajam.20200803.13
}

Received: March 26, 2020; Accepted: May 12, 2020; Published: May 27, 2020

\begin{abstract}
Investigating the heat transfer in aquifer thermal energy storage system is of interest since a deeper understanding of this phenomenon can be used to improve the behavior of a building, including relevant thermal parameters such as heating, cooling, and control systems. In this paper, we have presented a pair of coupled partial differential equations, which characterize the temperature distribution within the aquifer thermal energy storage system during the thermal injection process. The heat transfer equation is considered when the temperature difference between the solid and fluid phases is very small. We showed the solution to the model is positive and bounded. Simulations have been carried out for a constant Peclet number of 0.5, 500 and 100 . Hot water is considered being injected throughout the depth of a single injection well into the aquifer at one end of the domain and the temperature of the hot water is assumed to be constant throughout the whole injection period. The finite element method has been utilized to solve the governing equations numerically. The results showed that the temperature front of injected hot water passes through the aquifer from left to right and the temperature of the aquifer increases gradually with the passage of injection time. Furthermore, if the Peclet number is very high the temperature of injected hot water makes a high change on the aquifer temperature, while if Peclet number is less than 1 there is a little change on the aquifer temperature as time $t$ increases.
\end{abstract}

Keywords: Porous Media, Heat Transfer, Fluid Flow, Finite Element Method, Homogeneous Aquifer Thermal Energy Storage System

\section{Introduction}

Thermal energy storage systems around the world are often utilized to provide economical and environmental solutions to the energy problems [1]. Among various types and sizes of storage media, soil or underground aquifers, known as underground thermal energy storage are mostly used for seasonal heat/cold storage due to their large thermal capacity and thermal inertia [2]. One of the more common storage types among underground thermal energy storage systems is aquifer thermal energy storage system in which groundwater is used to carry the thermal energy into and out of an aquifer [3]. An aquifer [4] is an underground layer of water-bearing permeable rock, rock fractures or unconsolidated materials (gravel, sand, or silt) from which groundwater can be extracted using a water well.

Aquifer thermal energy storage system has become a wellknown energy storage technology around the world for the seasonal storage of heat and cold energy for heating and cooling buildings [1, 5-7]. Storage and recovery of thermal energy are achieved by extraction and injection of groundwater from aquifers using groundwater wells.

Aquifer thermal energy storage can serve as a costeffective technology to reduce the primary energy consumption of a building and associated $\mathrm{CO}_{2}$. One of the basic theories of this system is injecting the hot or cold water into an aquifer for long term storage and extracting that in the time of demand. An aquifer thermal energy storage system operates in a full cycle consisting of mainly four stages. The injection of hot/cold water into the aquifer, storage of the 
hot/cold water, production and the heating/cooling of buildings [8].

The use of aquifer for storing energy for long term started from the early 1970s. R. T. Rabbimov, G. Y. Umarov, R. A. Zakhidov [9], C. F. Meyer, D. K. Todd [10], did some early studies on this topic which mainly present analytical and semi-analytical solutions and economic considerations. Other analytical models and solutions on this topic were developed by Sauty J. P., Gringarten A. C., Menjoz A., Landel P. A. [11], Chen C.-S. D., Reddell L., [12], Voigt H. D., Haefner F. [13], Ziagos J. P., Blackwell D. D [14], Li K.-Y., Yang S.-Y., Yeh H.-D. [15], Yang S.-Y., Yeh H.-D. [16], which present solutions for idealistic systems under simplifying assumptions or qualitative estimations. Another analytical solution was developed by Bödvarsson G. S., Tsang C. F. [17], to investigate the movement of the cold water thermal front through subsurface media with equally spaced horizontal fractures.

Birhanu, Z. K., Kitterød, N.-O., Krogstad, H., Kværnø [23], presented analytical and numerical radial solutions of the differential equations for heat transport in water-saturated porous media and used analytical solutions to understand the quality of numerical simulations by doing simple numerical experiments. One of the experiments was an idealized aquifer thermal energy storage production sequence consisting of repeated injection and pumping of hot water in a confined aquifer. Another numerical model study was performed by Ganguly, S., Mohan Kumar, M. S., Date, A., Akbarzadeh [24], on a general 3D coupled thermo-hydro geological numerical model of an aquifer thermal energy storage system operating in cyclic mode to predict the 3D transient temperature distribution in the aquifer thermal energy storage system due to injection of hot water into the aquifer during summer and cold water in winter. They analyzed the movements of the hot water thermal front with time which is generated due to continuous injection.

Gao, L., Zhao, J., An, Q., Wang, J., Liu, X [25], reviewed system performance studies of aquifer thermal energy storage to facilitate the designers and users. Both the thermal performance and the economic and environmental performance were included. The thermal performance indicators and their definitions were summarized and influence factors were presented in their study. In another recent paper Ganguly, S., Kumar, M. S. M [26], developed a coupled thermo-hydro geological numerical model for an aquifer thermal energy storage system consisting of a confined porous aquifer underlain and overlain by impermeable rock media with different thermo-hydro geological properties. Ganguly, S., Tan, L., Date, A., Kumar, M [27] developed a coupled thermo-hydrogeological nodel for temperature distribution in a heterogeneous geothermal reservoir due to thermal injection. The objective of the present study is to develop a two dimensional numerical model for the transient temperature distribution due to thermal injection process in a homogeneous aquifer thermal energy storage system.

\section{Mathematical Model}

Let $\Omega \subset \mathfrak{R}^{2}$ be a bounded simply connected open domain in $\mathfrak{R}^{2}$ with a Lipchitz continuous boundary $\partial \Omega$. The governing partial differential equation of aquifer thermal energy storage model is given by the heat transfer and fluid flow equation that are coupled through the fluid velocity $q$ [18]. The system of equation is

$$
\begin{gathered}
S_{S} \frac{\partial h}{\partial t}+\nabla \cdot q=G, \\
\frac{\partial T}{\partial t}+\nabla(\mathrm{T} \cdot q)=\frac{1}{P e} \nabla^{2} T+H,
\end{gathered}
$$

where $h$ is a piezometric (hydraulic) head, $T$ is temperature and $q=-K \cdot \nabla h$ is the Darcy velocity. $K$ is hydraulic conductivity, $S_{S}$ is specific storage coefficient defined as the volume of water released from storage per unit change in head per unit volume of porous material, $G$ is volumetric flux per unit volume representing fluid injection and production and $H$ is an external heat source. Equation (1)-(2) must be completed by assigning an initial condition

$$
\begin{aligned}
& h(x, y, 0)=h_{0}(x, y),(x, y) \in \Omega, \\
& T(x, y, 0)=T_{0}(x, y),(x, y) \in \Omega,
\end{aligned}
$$

together with boundary conditions, which can take the form

$$
\begin{aligned}
& T(x, y, t)=p(x, y, t) \text { and } h(x, y, t)=q(x, y, t),(x, y) \in \\
& \qquad \partial \Omega_{\mathrm{D}}, \mathrm{t}>0, \\
& \frac{\partial T}{\partial n}=r(x, y, t) \text { and } \frac{\partial h}{\partial n}=s(x, y, t),(x, y) \in \partial \Omega_{\mathrm{N}}, \mathrm{t}>0,
\end{aligned}
$$

where $T_{0}, h_{0}, p, q, r$ and $s$ are given functions and $\left\{\partial \Omega_{\mathrm{D}}, \partial \Omega_{\mathrm{N}}\right\}$ provides a boundary partition. $\partial \Omega_{\mathrm{D}}$ is boundary condition and $\partial \Omega_{\mathrm{N}}$ is Neumann boundary condition.

The problem cannot be formulated in terms of second derivatives (which may not exist throughout the solution space), and an integral form is used in which the solution belongs to the Sobolev space $H^{1}(\Omega)$ with the underlying space being $L^{2}(\Omega) . L^{2}(\Omega)$ is defined as [19]

$$
L^{2}(\Omega)=\left\{v: \int_{\Omega}|v(x, y)|^{2} d \Omega<\infty\right\},
$$

and the Sobolov space $H^{1}(\Omega)$ as

$$
H^{1}(\Omega)=\left\{v \in L^{2}(\Omega): \frac{\partial v}{\partial x} \in L^{2}(\Omega), \frac{\partial v}{\partial y} \in L^{2}(\Omega)\right\},
$$

equipped with the norm

$$
\|v\|_{H^{1}(\Omega)}=\left\{\sum|v|_{H^{1}(\Omega)}^{2}\right\}^{\frac{1}{2}} .
$$

For vanishing boundary values, we define

$$
H_{0}^{1}(\Omega)=\left\{v \in H^{1}(\Omega):\left.v\right|_{\partial \Omega_{\mathrm{D}}}=p(x, y)\right\} .
$$

We shall often use Sobolev's embedding, for any real number $p \geq 1$, there exist constants $S_{p}$ and $S^{0}{ }_{p}$ such that 


$$
\forall v \in H^{1}(\Omega),\|v\|_{L^{p}(\Omega)} \geq S_{p}\|v\|_{H^{1}(\Omega)}
$$

and

$$
\forall v \in H^{1}{ }_{0}(\Omega),\|v\|_{L^{p}(\Omega)} \geq S^{0}{ }_{p}\|v\|_{H^{1}(\Omega)} .
$$

When $p=2$, then it reduces to Poincare inequality.

\section{Finite Element Method}

To solve the system of the partial differential equation (1)(2) numerically using finite element method, we will introduce a weak formulation and proceed formally, by multiplying for each $t>0$ by a test function $v=v(x, y)$ and integrating on $\Omega$. We set $V=H^{1}(\Omega)$ and for each $t>0$ we seek $h(t), T(t) \in V$ such that

$$
\int_{\Omega}\left(S_{s} \frac{\partial h}{\partial t} v d \Omega+\nabla \cdot q v\right) d \Omega=\int_{\Omega} G v d \Omega
$$

$\int_{\Omega} \frac{\partial T}{\partial t} v d \Omega+\int_{\Omega} \nabla(\mathrm{T} \cdot q) v d \Omega=\frac{1}{P e} \int_{\Omega}^{\cdot} \nabla^{2} T v d \Omega+\int_{\Omega} H v d \Omega$,

By putting $q=-K \cdot \nabla h$ into (3)-(4) and applying the Green's theorem stated in [20] we have

$$
\begin{aligned}
S_{s} \int_{\Omega} \frac{\partial h}{\partial t} v d \Omega+\mathrm{a}(\mathrm{h}, \mathrm{v}) & =\int_{\Omega} G v d \Omega+\int_{\partial \Omega}^{\cdot} v \frac{\partial h}{\partial n} d s, \\
\int_{\Omega} \frac{\partial T}{\partial t} v d \Omega+\mathrm{b}(\mathrm{T}, \mathrm{v}) & =\int_{\Omega} \operatorname{Hvd} \Omega+\int_{\partial \Omega} v \frac{\partial T}{\partial n} d s,
\end{aligned}
$$

where

$$
a(h, v)=\int_{\Omega} K \nabla h \nabla v d \Omega
$$

$b(T, v)=\int_{\Omega} q \nabla T v d \Omega+\frac{1}{P e} \int_{\Omega} \nabla T \nabla v d \Omega \quad$ with $\quad T(0)=T_{0}$ and $h(0)=h_{0}$. Writing $\langle.,$.$\rangle for the L^{2}(\Omega)$ inner product we may write the weak formulation (5)-(6) as

$$
\begin{aligned}
& \left\langle\frac{\partial h}{\partial t}, v\right\rangle+\mathrm{a}(\mathrm{h}, \mathrm{v})=\langle G, v\rangle+\int_{\partial \Omega} v \frac{\partial h}{\partial n} d s, \\
& \left\langle\frac{\partial T}{\partial t}, v\right\rangle+\mathrm{b}(\mathrm{T}, \mathrm{v})=\langle H, v\rangle+\int_{\partial \Omega} v \frac{\partial T}{\partial n} d s .
\end{aligned}
$$

We now consider the Galerkin approximation of problem (7)-(8) for each $t>0$ and let $V_{l}$ be the space of Lagrange finite elements of degree $r$ vanishing on the boundary with respect to a mesh of $\Omega$ of mesh size $l$, and define $h_{l}$ and $T_{l}$ to be the finite element solution, $h_{l}, T_{l} \in V_{l}$ such that

$$
\left\langle\frac{\partial h_{l}}{\partial t}, v_{l}\right\rangle+\mathrm{a}\left(h_{l}, v_{l}\right)=\left\langle G, v_{l}\right\rangle+\int_{\partial \Omega} v_{l} \frac{\partial h_{l}}{\partial n} d s,(9)
$$$$
\left\langle\frac{\partial T_{l}}{\partial t}, v_{l}\right\rangle+\mathrm{b}\left(T_{l}, v_{l}\right)=\left\langle H, v_{l}\right\rangle+\int_{\partial \Omega} v_{l} \frac{\partial T_{l}}{\partial n} d s
$$

with $T_{l}(0)=T_{0 l}$ and $h_{l}(0)=h_{0 l}$ where $V_{l} \subset V$ is a suitable space of a finite dimension and $T_{0 l}$ and $h_{0 l}$ is a convenient approximation of $T_{0}$ and $h_{0}$ in the space $V_{l}$ respectively.

\subsection{Existence and Uniqueness}

Since $H^{1}(\Omega)$ is separable, therefore, it has a countable basis $\left\{\phi_{i}\right\}_{i \geq 1}$ Let $V_{l}$ be space spanned by the first $l$ basis functions, $\left\{\phi_{i}\right\}_{1 \leq i \leq l}$. The reduced problem (10) is discretized in $V_{m}$ by the square system of equations. That is finding $T_{l}=\sum_{1 \leq i \leq l} T_{i} \phi_{i} \in V_{l}$ solution of

$$
\left\langle\frac{\partial T_{l}}{\partial t}, v_{l}\right\rangle+\mathrm{b}\left(T_{l}, v_{l}\right)=\left\langle H, v_{l}\right\rangle+\int_{\partial \Omega} v_{l} \frac{\partial T_{l}}{\partial n} d s,\left\langle T_{l}(0), v_{l}\right\rangle=\left\langle T_{0 l}, v_{l}\right\rangle .
$$

The guarantee for existence and uniqueness of the solution of the weak formulation equation (8) is Picard-Lindelof Theorem which is stated in [21] as:

Theorem 1. Picard-Lindelof Theorem: Let $V$ and $H$ be separable Hilbert spaces with $V \subset H, V$ dense in $H$ and $\|v\|=$ $c\|v\|_{V}$. Assume that there are constants $\mu_{1} \geq \mu_{2}>0$ with

$$
b(v, v)=\mu_{2}\|v\|_{V}^{2}, \forall v \in V
$$

and

$$
|b(w, v)| \leq \mu_{2}\|w\|_{V}\|v\|_{V}, \forall v, w \in V
$$

Then there exists a unique solution $T \in L^{2}\left(\left(0, T_{f}\right), V\right)$ of the initial value problem

$$
\left\langle\frac{\partial T(t)}{\partial t}, v\right\rangle+b(T(t), v)=\langle H, v\rangle, T(0)=T_{0}, v \in V .
$$

Condition (12) is often referred to as coercivity or positivity, while (13) is called continuity of boundedness.

Further, let $b(w, v)=c(w, v)+d(w, v) \quad$ where $c(w, v)=\frac{1}{P e} \int_{\Omega} \nabla w \nabla v d \Omega$ and $d(w, v)=\int_{\Omega} q \nabla w v d \Omega$. Now, we went to check whether our problem satisfies condition (12) and (13).

Coercivity/Positivity

To ensure that $b(v, v)$ is positive we must put some restriction on the flow velocities. That is we need

$$
|d(v, v)| \leq|b(v, v)|
$$

and assume the bounded velocities $\|q\|_{\infty}$. Using Poincare inequality $\|v\|_{H^{1}{ }_{0}} \leq C_{\Omega}\|v\|_{H^{1}}$ where $C_{\Omega}$ is some constant [21].

$$
\text { Now if } C_{\Omega}\|q\|_{\infty} \leq D \frac{1}{P e} \text { with } D<1 \text { we obtain }
$$

$$
\begin{aligned}
& b(v, v)=\int_{\Omega} \frac{1}{P e} \nabla v \nabla v d \Omega+\int_{\Omega} q \nabla v v d \Omega \geq \frac{1}{P e}\|v\|_{H^{1}}\|v\|_{H^{1}}-\|q\|_{\infty}\|v\|_{H^{1}}\|v\|_{H_{0}} \geq\left(\frac{1}{P e}-C_{\Omega}\|q\|_{\infty}\right)\|v\|_{H^{1}}\|v\|_{H^{1}} \\
& \geq\left(\frac{1}{P e}(1-D)\right)\|v\|_{H^{1}}^{2} \geq \mu_{1}\|v\|_{H^{1}}^{2}
\end{aligned}
$$


since $D<1$, hence $\mu_{1}=\frac{1}{P e}(1-D)>0$. Therefore, $b(v, v) \geq \mu_{1}\|v\|_{H^{1}}^{2}$. Hence positivity condition is satisfied.

Continuity/Boundedness

The second condition the boundedness follows by applying Cauchy Schwartz inequality, $b(w, v)=$ $\int_{\Omega} \frac{1}{P e} \nabla w \nabla v d \Omega+\int_{\Omega} q \nabla w v d \Omega \leq \frac{1}{P e}\|w\|_{H^{1}}\|v\|_{H^{1}}+$ $\|q\|_{\infty}\|v\|_{H^{1}}\|v\|_{H_{0}{ }^{1}} \leq\left(\frac{1}{P e}+C_{\Omega}\|q\|_{\infty}\right)\|w\|_{H^{1}}\|v\|_{H^{1}} \geq$ $\mu_{2}\|w\|_{H^{1}}\|v\|_{H^{1}}$,

where $\mu_{2}=\left(\frac{1}{P e}+C_{\Omega}\|q\|_{\infty}\right)>0$. Hence continuity condition satisfied. Therefore, the solution of the weak formulation of our problem exists and unique.

\subsection{Approximate Solution}

let $e$ denotes the element number in a region $\Omega$. To provide an algebraic interpretation of (9)-(10) we introduce a basis $\phi_{j}$ for $V_{l}$, and we observe that it suffices that (9)-(10) are verified for the basis functions to be satisfied by all the functions of the subspace. Moreover, as for each $t>0$, the solution to the finite element problem belongs to the subspace as well, we will have

$$
h_{l}^{e}(x, y, t)=\sum_{j=1}^{N_{l}} h_{j}^{e}(t) \phi_{j}(x, y)
$$

and

$$
T_{l}^{e}(x, y, t)=\sum_{j=1}^{N_{l}} T_{j}^{e}(t) \phi_{j}(x, y),
$$

where the $h_{j}^{e}(t)$ and $T_{j}^{e}(t)$ coefficients represent the unknowns of the problem (9)-(10) on the element $e$ and $N_{l}=3$, since the elements are triangular.

Denoting by $\dot{h}_{j}{ }^{e}(t)$ and $\dot{T}_{j}{ }^{e}(t)$ the derivatives of the function $h_{j}{ }^{e}(t)$ and $T_{j}{ }^{e}(t)$ respectively with respect to time, a system of equation (9)-(10) on the element $e$ becomes

$$
\begin{gathered}
\int_{\Omega^{\mathrm{e}}} \sum_{j=1}^{N_{l}} S_{s} \dot{h}_{j}{ }^{e}(t) \phi_{\mathrm{j}} \phi_{\mathrm{i}} \mathrm{d} \Omega^{\mathrm{e}}+\mathrm{a}\left(\sum_{\mathrm{j}=1}^{N_{l}} \dot{h}_{j}^{e}(t) \phi_{\mathrm{j}} \phi_{\mathrm{i}} \mathrm{d} \Omega^{\mathrm{e}}\right)=\int_{\Omega^{\mathrm{e}}} G \phi_{\mathrm{i}} \mathrm{d} \Omega^{\mathrm{e}}+\int_{\partial \Omega^{\mathrm{e}}} \phi_{\mathrm{i}} \mathrm{s}(\mathrm{x}, \mathrm{y}) \mathrm{ds}, \mathrm{i}=1,2,3, \ldots, N_{l}, \\
\int_{\Omega^{\mathrm{e}}} \sum_{j=1}^{N_{l}} \dot{T}_{j}^{e}(t) \phi_{\mathrm{j}} \phi_{\mathrm{i}} \mathrm{d} \Omega^{\mathrm{e}}+\mathrm{b}\left(\sum_{\mathrm{j}=1}^{N_{l}} \dot{T}_{j}^{e}(t) \phi_{\mathrm{j}} \phi_{\mathrm{i}} \mathrm{d} \Omega^{\mathrm{e}}\right)=\int_{\Omega^{\mathrm{e}}} H \phi_{\mathrm{i}} \mathrm{d} \Omega^{\mathrm{e}}+\int_{\partial \Omega^{\mathrm{e}}} \phi_{\mathrm{i}} \mathrm{r}(\mathrm{x}, \mathrm{y}) \mathrm{ds}, \mathrm{i}=1,2,3, \ldots, N_{l} .
\end{gathered}
$$

Then after some implementation, the system can be rewritten in the form

$$
\begin{aligned}
& S_{s} M^{e} \dot{h}^{e}+A^{e} h^{e}=g^{e} \\
& M^{e} \dot{T}^{e}+B^{e} T^{e}=r^{e}
\end{aligned}
$$

where

1. $M^{e}, A^{e}$ and $B^{e} 3 \times 3$ matrix whose elements are given below respectively

$$
\begin{gathered}
m^{e}{ }_{j i}=\int_{\Omega^{\mathrm{e}}} \phi_{j} \phi_{i} d \Omega^{e}, a^{e}{ }_{j i}=\int_{\Omega^{\mathrm{e}}} \boldsymbol{K} \nabla \phi_{j} \nabla \phi_{i} d \Omega^{e}, \\
b^{e}{ }_{j i}=\int_{\Omega^{\mathrm{e}}} \boldsymbol{q} \nabla \phi_{j} \phi_{i} d \Omega^{e}+\frac{1}{P e} \int_{\Omega^{\mathrm{e}}} \nabla \phi_{j} \nabla \phi_{i} d \Omega^{e} .
\end{gathered}
$$

2. $g^{e}$ and $r^{e}$ column vector whose elements are given below respectively

$$
\begin{aligned}
& g_{i}^{e}=\int_{\Omega^{\mathrm{e}}} G \phi_{\mathrm{i}} \mathrm{d} \Omega^{\mathrm{e}}+\int_{\partial \Omega^{\mathrm{e}}} \phi_{\mathrm{i}} \mathrm{s}(\mathrm{x}, \mathrm{y}) \mathrm{ds}, \\
& r^{e}{ }_{i}=\int_{\Omega^{\mathrm{e}}} H \phi_{\mathrm{i}} \mathrm{d} \Omega^{\mathrm{e}}+\int_{\partial \Omega^{\mathrm{e}}} \phi_{\mathrm{i}} \mathrm{r}(\mathrm{x}, \mathrm{y}) \mathrm{ds},
\end{aligned}
$$

The matrix $A^{e}$ is called the local stiffness matrix and $M^{e}$ is called the local mass matrix. Now by assembling process
(14)-(15) will be used to get the overall approximate solution and after some algebraic manipulation, which will follow shortly, allows to be put into the form

$$
\begin{gathered}
S_{s} M \dot{h}+A h=g, \\
M \dot{T}+B T=r,
\end{gathered}
$$

in which $h=\left[h_{1}, h_{2}, \ldots, h_{N}\right]^{T}, T=\left[T_{1}, T_{2}, \ldots, T_{N}\right]^{T}$ and the $N \times N$ matrix $M$ is a global mass matrix, the $N \times N$ matrix $A$ is the global stiffness matrix, and the vector $\boldsymbol{g}$ and $\boldsymbol{r}$ are the globalized force vector. Here $N$ denotes the total number of nodes in the problem as a whole, and the components of $h$ and $T$ are now labelled by their global node numbers. For the numerical solution of the ODE system (16)-(17), many methods are available from that we have observed backward Euler method of the form

$$
\begin{gathered}
S_{S} M \frac{h^{k+1}-h^{k}}{\Delta t}+A h^{k+1}=g^{k+1}, \\
M \frac{T^{k+1}-T^{k}}{\Delta t}+B T^{k+1}=r^{k+1},
\end{gathered}
$$

which is first order accurate with respect to $\Delta t=t^{k+1}-t^{k}$.

\section{Error Analysis}

\subsection{Prior Error Analysis}

Consider the weak formulation of the governing equation 
(10) of heat transfer in porous media. Before analyzing a weak formulation (10), we analyze its convergence, since it is less involved. The key to the analysis of the weak formulation is to compare $T_{l}$ not directly to $T$, but rather to an appropriate representative $w_{l} \in C^{1}\left(\left[0, T_{f}\right], V_{l}\right)$. For $w_{l}$ we choose the elliptic projection of $T$, defined by

$$
b\left(w_{l}, v\right)=b(T, v), v \in V_{l} 0 \leq t \leq T_{f}
$$

From [22] the finite element method for elliptic problems, we have the $L^{2}$ estimate

$$
\left\|T(t)-w_{l}(t)\right\| \leq p l^{r+1}\|T(t)\|_{r+1}, 0 \leq t \leq T_{f}
$$

for some constant $p$. If we differentiate (20), we see that $\frac{\partial w_{l}}{\partial t}$ is the elliptic projection of $\frac{\partial T}{\partial t}$,

so

$$
\left\|\frac{\partial T}{\partial t}(t)-\frac{\partial w_{l}}{\partial t}(t)\right\| \leq p l^{r+1}\left\|\frac{\partial T}{\partial t}(t)\right\|_{r+1}, 0 \leq t \leq T_{f}
$$

Now,

$$
\left\langle\frac{\partial w_{l}}{\partial t}, v\right\rangle+b\left(w_{l}, v\right)=\left\langle\frac{\partial w_{l}}{\partial t}, v\right\rangle+b(T, v)=\left\langle\frac{\partial\left(w_{l}-T\right)}{\partial t}, v\right\rangle+
$$$$
\langle H, v\rangle, v \in V_{l}, 0 \leq t \leq T_{f} .
$$

Let $y_{l}=w_{l}-T_{l}$. Subtracting (10) from (23), we get

$$
\left\langle\frac{\partial y_{l}}{\partial t}, v\right\rangle+b\left(y_{l}, v\right)=\left\langle\frac{\partial\left(w_{l}-T\right)}{\partial t}, v\right\rangle, v \in V_{l}, 0 \leq t \leq T_{f} .
$$

Now, for each $t$ we choose $v=y_{l}(t) \in V_{l}$. Note that for any function $y \in C^{1}\left([0, T] ; L^{2}(\Omega)\right)$,

$$
\|y\| \frac{d}{d t}\|y\|=\frac{1}{2} \frac{d}{d t}\|y\|^{2}=\left\langle\frac{\partial y}{\partial t}, y\right\rangle .
$$

Thus we obtain

$$
\left\|y_{l}\right\| \frac{d}{d t}\left\|y_{l}\right\|+b\left(y_{l}, y_{l}\right)=\left\langle\frac{\partial\left(w_{l}-T\right)}{\partial t}, y_{l}\right\rangle \leq\left\|\frac{\partial\left(w_{l}-T\right)}{\partial t}\right\|\left\|y_{l}\right\|,
$$

cancelling the same expression on both sides of (24) we obtain

$$
\frac{d}{d t}\left\|y_{l}\right\| \leq\left\|\frac{\partial\left(w_{l}-T\right)}{\partial t}\right\| \leq p l^{r+1}\left\|\frac{\partial T}{\partial t}(t)\right\|_{r+1} .
$$

This holds for each $t$. Integrating over $[0, t]$, we get

$$
\begin{aligned}
& \left\|y_{l}(t)\right\| \leq \|\left(w_{l}(t)-T(t) \|\right. \\
& \quad \leq\left\|y_{l}(0)\right\|+p l^{r+1}\|T(t)\|_{L^{1}\left([0, T] ; H^{r+1}(\Omega)\right)} .
\end{aligned}
$$

For $y_{l}(0)$ we have

$$
\begin{gathered}
\left\|y_{l}(0)\right\| \leq\left\|w_{l}(0)-T_{l}(0)\right\| \leq\left\|w_{l}(t)-T(0)\right\|+\left\|T_{0}-T_{l}(0)\right\| \\
\leq p l^{r+1}\left\|T_{0}\right\|_{r+1}+\left\|T_{0}-T_{l}(0)\right\|
\end{gathered}
$$

Thus, assuming that the exact solution is sufficiently smooth and the initial data $T_{l}(0)$ is chosen so that $\| T_{0}-$ $T_{l}(0) \|=O\left(l^{r+1}\right)$, we have

$$
\left\|y_{l}\right\|_{L^{\infty}\left([0, T] ; L^{2}(\Omega)\right)}=O\left(l^{r+1}\right) .
$$

Combining this estimate with the elliptic estimate (21) we get an estimate on the error

$$
\left\|T-T_{l}\right\|_{L^{\infty}\left([0, T] ; L^{2}(\Omega)\right)}=O\left(l^{r+1}\right) .
$$

\subsection{Posterior Error Estimate}

Now we turn to the error analysis of a fully discrete scheme, which is finite elements in space and backward Euler in time. Writing $T_{l}^{j}$ for $T_{l}(\cdot, j k)$ (with $k$ the time step), the scheme is

$$
\begin{gathered}
\left\langle\frac{T_{l}^{j+1}-T_{l}{ }^{j}}{k}, v\right\rangle+b\left(T_{l}^{j+1}, v\right)=\left\langle H^{j+1}, v\right\rangle, v \in V_{l}, j= \\
0,1,2, \ldots, M .
\end{gathered}
$$

To analyze this scheme, we proceed as we did for the semidiscrete scheme, with some extra complications coming from the time discretization. In particular, we continue to use the elliptic projection $w_{l}$ as a representative of $T$. Thus the consistency error is given by

$$
\begin{gathered}
E=\left\langle\frac{w_{l}{ }^{j+1}-w_{l}{ }^{j}}{k}, v\right\rangle+b\left(w_{l}{ }^{j+1}, v\right)-\left\langle H^{j+1}, v\right\rangle \\
=\left\langle\frac{T^{j+1}-T^{j}}{k}, v\right\rangle+b\left(T^{j+1}, v\right)-\left\langle H^{j+1}, v\right\rangle \\
+\left\langle\frac{\left(w_{l}^{j+1}-T^{j+1}\right)-\left(w_{l}^{j}-T^{j}\right)}{k}, v\right\rangle \\
=\left\langle\frac{T^{j+1}-T^{j}}{k}-\frac{\partial T^{j+1}}{\partial t}, v\right\rangle+\left\langle\frac{\left(w_{l}^{j+1}-T^{j+1}\right)-\left(w_{l}^{j}-T^{j}\right)}{k}, v\right\rangle \\
=\left\langle z^{j}, v\right\rangle,
\end{gathered}
$$

where the last line defines $z^{j}$. Next, we estimate the two terms that comprise $z^{j}$, in $L^{2}$. First, we have

$$
\left\|\frac{T^{j+1}-T^{j}}{k}-\frac{\partial T^{j+1}}{\partial t}\right\| \leq \frac{k}{2}\left\|\frac{\partial^{2} T}{\partial t^{2}}\right\|_{L^{\infty}\left(L^{2}\right)},
$$

by Taylor's theorem. Next,

$$
\begin{aligned}
& \frac{\left(w_{l}^{j+1}-T^{j+1}\right)-\left(w_{l}^{j}-T^{j}\right)}{k} \\
& =\frac{1}{k} \int_{j k}^{(j+1) k} \frac{\partial}{\partial t}\left[w_{l}(s)-T(s)\right] d s,
\end{aligned}
$$

so

$$
\begin{aligned}
& \left\|\frac{\left(w_{l}^{j+1}-T^{j+1}\right)-\left(w_{l}^{j}-T^{j}\right)}{k}\right\| \\
& =p l^{r+1}\left\|\frac{\partial T}{\partial t}\right\|_{L^{\infty}\left([j k,(j+1) k], H^{r+1}(\Omega)\right)}
\end{aligned} .
$$

Thus we have obtained abound on the consistency error: 


$$
E=\left\langle\frac{w_{l}{ }^{j+1}-w_{l}{ }^{j}}{k}, v\right\rangle+b\left(w_{l}{ }^{j+1}, v\right)-\left\langle H^{j+1}, v\right\rangle=\left\langle z^{j}, v\right\rangle, v
$$

with

$$
\begin{aligned}
\left\|z^{j}\right\| & =p\left(k\left\|\frac{\partial^{2} T}{\partial t^{2}}\right\|_{L^{\infty}\left(\left[0, T_{f}\right], H^{r+1}(\Omega)\right)}\right) \\
& \left.+l^{r+1}\left\|\frac{\partial T}{\partial t}\right\|_{L^{\infty}\left(\left[0, T_{f}\right], H^{r+1}(\Omega)\right)}\right) .
\end{aligned}
$$
$\left.T_{l}\right)$,

Combining with the scheme (25), we get (for $y_{l}=w_{l}$ -

$$
\left\langle\frac{y_{l}^{j+1}-y_{l}^{j}}{k}, v\right\rangle+b\left(y_{l}{ }^{j+1}, v\right)=\left\langle z^{j}, v\right\rangle, v \in V_{l} .
$$

We conclude the argument with a stability argument. Choose $v=y_{l}{ }^{j+1} \in V_{l}$. This becomes:

$$
\left\|y_{l}{ }^{j+1}\right\|^{2}+k b\left(y_{l}^{j+1}, y_{l}^{j+1}\right)=\left\langle y_{l}^{j}+k z^{j}, y_{l}^{j+1}\right\rangle,
$$

so

$$
\left\|y_{l}{ }^{j+1}\right\| \leq\left\|y_{l}^{j}\right\|+k E, j=0,1,2, \ldots, M,
$$

and, by iteration,

$$
\max _{0 \leq j \leq M}\left\|y_{l}{ }^{j}\right\| \leq\left\|y_{l}{ }^{0}\right\|+T E, j=0,1,2, \ldots, M,
$$

where $T=k M$ and $E$ is consistency error. In this way, we prove that

$$
\max _{0 \leq j \leq M}\left\|T^{j}-T_{l}^{j}\right\|=O\left(k+l^{r+1}\right), j=0,1,2, \ldots, M .
$$

\section{Numerical Results and Discussion}

In this section, we demonstrate the numerical results of heat transfer in aquifer thermal energy storage due to hot water injection. We assume the governing equation of Aquifer Thermal Energy Storage model as local thermal equilibrium which states the temperature of each phase present in a representative elementary volume equals to the average temperature of the representative fixed control volume element. We also assume no internal sinks or sources. Moreover, we assume the aquifer to be homogeneous and isotropic, thus $K$ (hydraulic conductivity) has a constant scalar value. Consider the depth and width of the aquifer are to be constant and equal to 1 .

Hot water is considered being injected throughout the depth of a single injection well into the aquifer at one end of the domain. Assume the bottom of the aquifer is given to be impermeable and there is no infiltration through the top boundary. The temperature of the hot water is assumed to be $300^{\circ} \mathrm{K}$ which is kept constant throughout the whole injection period. The initial temperature of the aquifer water before the injection is assumed to be $273^{\circ} \mathrm{K}$. A fixed injection water temperature of $300^{\circ} \mathrm{K}$ is considered as a boundary condition at injection end and initial aquifer temperature of $273^{\circ} \mathrm{K}$ is taken as the second boundary condition at a long distance away from the injection point. The top and bottom boundaries are considered as fixed temperature boundaries with the temperature of both top and bottom boundary are equals to $273^{0} \mathrm{~K}$.

Assume the hot water is injected into the well with rate $Q$ through the screen located between $x_{a}$ and $x_{b}$ as shown in figure 1 . We consider the boundary condition and initial condition as $h=1$ and at the well (boundary) $x=0$ we consider

$$
\frac{\partial h}{\partial y}=\left\{\begin{array}{c}
-q x_{a} \leq x \leq x_{b} \\
0, \text { otherwise }
\end{array}\right.
$$

where the flux is $q=\frac{Q}{x_{a}-x_{b}}$.

The whole system is schematically presented in figure 1 . The numerical simulations of the study have been performed using the finite element method.

In this experiment for simplicity, we set fluid and solid heat capacities as $1 \mathrm{~J} /(\mathrm{kg} \cdot \mathrm{K})$, thermal conductivities are equal to $1 W /(m \cdot K)$ and the specific storage $S_{s}=1 / m$. For all the numerical results the well screen is located between $x_{a}=0.6667$ and $x_{b}=0.3333$.

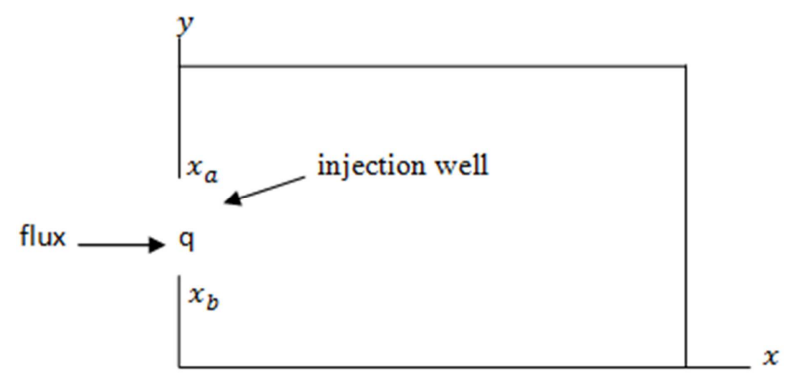

Figure 1. Schematic diagram of the homogeneous porous medium due to an injection well installed.

By real-life experiences, if heat is applied to the material with low thermal conductivity, the expected result would be that the heat concentrates at the applied spot and doesn't spread out very far throughout the specimen. As shown in figure 2, we can see that heat concentrates highly at the left side of the injection well (highest of 300 degrees between the well screen) and spreading to the right.

In Figures 3 and 4 we can see how the temperature front of injected hot water passes through the aquifer from left to right. The plot shows that continuous injection of hot water into cold aquifer environment a thermal interface or the thermal front is set up which propagates through the aquifer with time. 


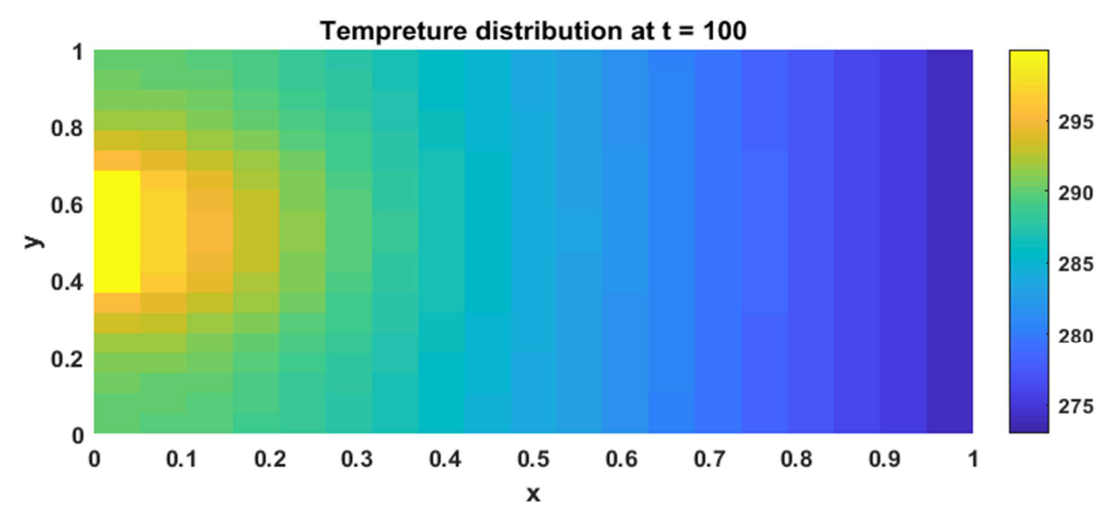

Figure 2. The temperature distribution at $t=100$.

The temperature of the aquifer also increases gradually with the passage of injection time due to the advancement of the thermal front. As the thermal front enters and enters in a homogeneous aquifer, a change in trend in the temperature

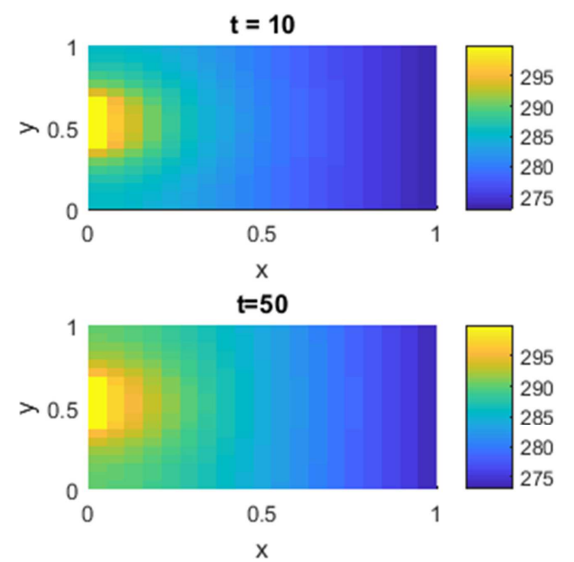

distribution is observed and sharp points are noticed on the temperature distribution plot. Also, as time increases the temperature of porous aquifer increase.
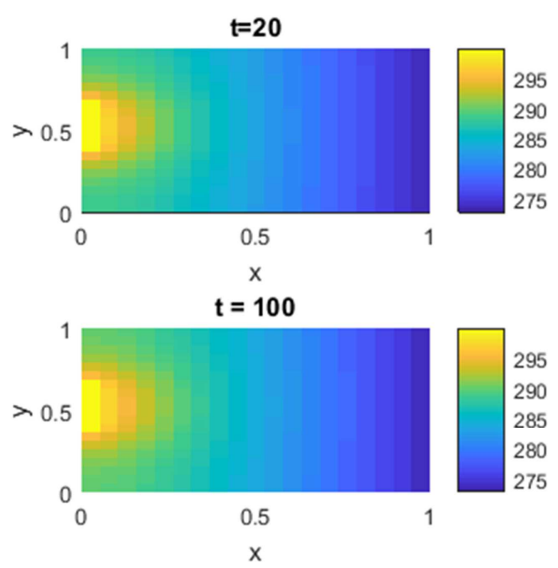

Figure 3. The temperature distribution in the aquifer for the different final time. Plots correspond to Peclet number of 50.

Figure 3 illustrates the heat flow when the heat transfer by the motion of a fluid (advection) has a stronger influence on the behaviour of a heat expansion than molecular motion (conduction). In this case, we set a Peclet number equals 50. Figure 4, on the contrary, shows the solution of the heat transfer equation when conduction dominates over convection, here the Peclet number equals to 0.5. The conductive term in equation (2) dominates when the Peclet

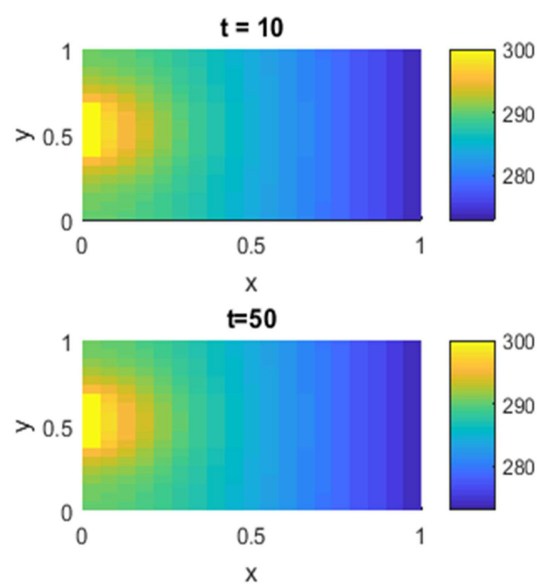

number is less than 1 . If the value of the Peclet number is very high, then advection strongly dominates over conduction or diffusion see figure 5 for Peclet number equal to 100 . This shows if the Peclet number is very high the temperature of injected hot water makes a high change on the aquifer temperature as time $t$ increase, while if Peclet number is less than 1 there is a little bit change on the aquifer temperature.

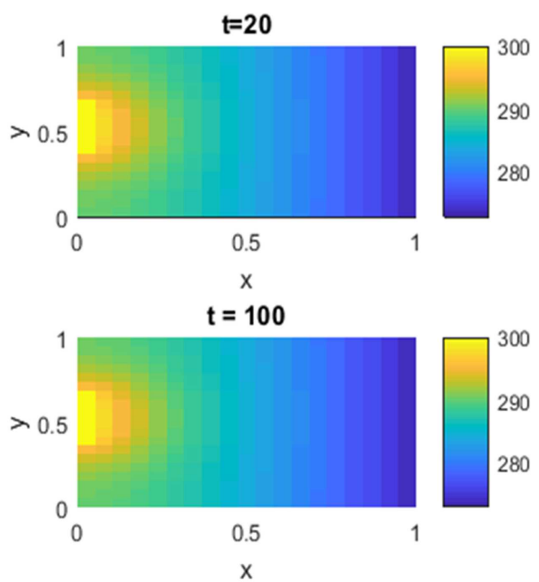

Figure 4. The temperature distribution in the aquifer for a different final time. Plots correspond to a Peclet number of 0.5. 

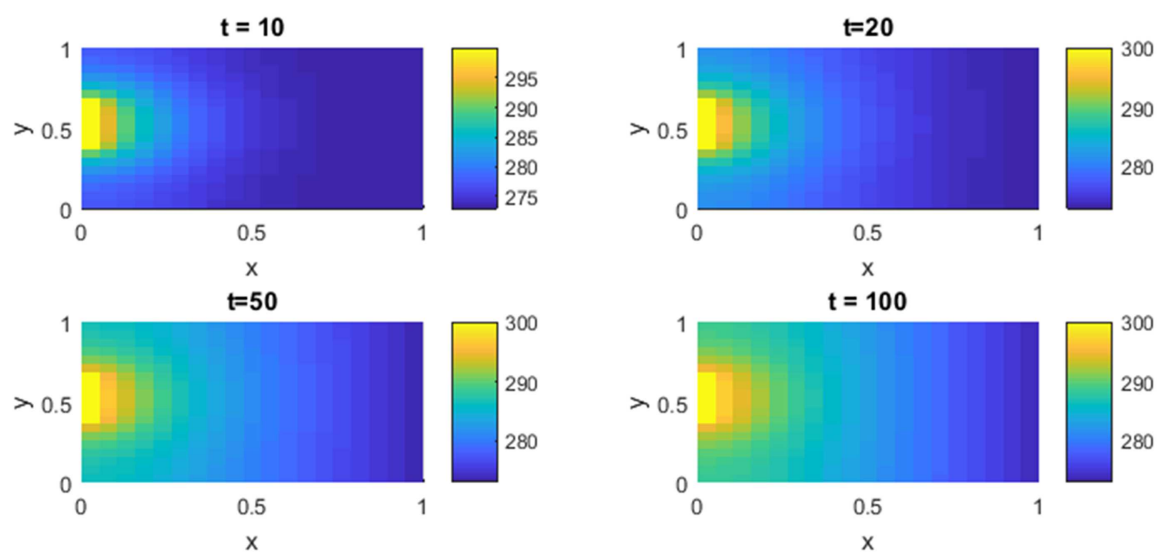

Figure 5. The temperature distribution in the aquifer for a different final time. Plots correspond to Peclet number of 100.

\section{Conclusion}

A two dimensional numerical model for heat transport in the aquifer thermal energy storage system during hot water injection in a single wall was presented considering constant injection rate. The primary target was to model the movement of the thermal front with time, which is generated in the aquifer due to the thermal injection. In general, we conclude from our numerical results that with the injection of hot water in a single wall thermal front are set up in the aquifer and proceeds with injection time. The aquifer temperature thus increases gradually with time at a fixed distance. The present two-dimensional models give some insight into the problem of transient heat transport phenomenon in a homogeneous porous aquifer due to hot water injection into it.

The advective flux of heat transfer is proportional to the volumetric injection rate of hot water to in an advection dominated system like the present one since the advective velocity of groundwater flow enhances due to increased injection rate. The results of the simulation give some preliminary results on the performance of aquifer thermal energy storage and their use in underground energy storage. Thus fluctuation of temperature could be seen during the injection of hot water in a homogeneous porous aquifer.

The heat loss from the aquifer plays a crucial role in the transient heat transport phenomenon in an aquifer thermal energy storage system. Due to heat loss, the advancement of the thermal front is always lesser in an aquifer with heat loss than that without it. Large heat loss from an aquifer makes it inefficient for the usage of long term thermal energy storage.

\section{References}

[1] H. O. Paksoy, Z. Gürbüz, B. Turgut, D. Dikici, H. Evliya, Aquifer thermal storage (ATES) for air-conditioning of a supermarket in Turkey, Renew. Energy. 29 (2004) 1991-1996.

[2] K. S. Lee, S. J. Jeong, Numerical modelling on the performance of aquifer thermal energy storage system under cyclic flow regime, Int. J. Green Energy. 5 (2008) 1-14.

[3] O. Andersson, Aquifer thermal energy storage (ATES), in Therm. Energy Storage Sustain. Energy Consum., Springer,
2007: pp. $155-176$.

[4] A. Vandenbohede, Solute transport in heterogeneous aquifers parameter identification and its use in groundwater pollution and salt water intrusion problems, (2004).

[5] T. Probert, G. Hellsröm, J. Glaesson, Thermohydraulic evaluation of two ATES projects in southern Sweden, in: Proc. Int. Symp. Aquifer Therm. Energy Storage, Tuscaloosa, AL, USA, 1994: pp. 73-81.

[6] H. O. Paksoy, O. Andersson, S. Abaci, H. Evliya, B. Turgut, Heating and cooling of a hospital using solar energy coupled with seasonal thermal energy storage in an aquifer, Renew. Energy. 19 (2000) 117-122.

[7] D. M. Allen, M. M. Ghomshei, T. L. Sadler-Brown, A. Dakin, D. Holtz, The current status of geothermal exploration and development in Canada, in: Proc. from World Geotherm. Congr., 2000: pp. 55-58.

[8] S. Dharma, Modeling of aquifer thermal energy storage (ATES) using heat and solute transport in 3D (HST3D), Civ. Eng. Dimens. 11 (2009) 119-126.

[9] R. T. Rabbimov, G. Y. Umarov, R. A. Zakhidov, Storage of solar energy in a sandy-gravel ground, Appl. Sol. Energy (USSR)(Engl. Transl.);(United States). 7 (1971).

[10] C. F. Meyer, D. K. Todd, Conserving energy with heat storage wells, Environ. Sci. Technol. 7 (1973) 512-516.

[11] J. P. Sauty, A. C. Gringarten, A. Menjoz, P. A. Landel, Sensible energy storage in aquifers: 1. A theoretical study, Water Resour. Res. 18 (1982) 245-252.

[12] C.-S. Chen, D. L. Reddell, Temperature distribution around a well during thermal injection and a graphical technique for evaluating aquifer thermal properties, Water Resour. Res. 19 (1983) 351-363.

[13] H. D. Voigt, F. Haefner, Heat transfer in aquifers with finite caprock thickness during a thermal injection process, Water Resour. Res. 23 (1987) 2286-2292.

[14] J. P. Ziagos, D. D. Blackwell, A model for the transient temperature effects of horizontal fluid flow in geothermal systems, J. Volcanol. Geotherm. Res. 27 (1986) 371-397.

[15] K.-Y. Li, S.-Y. Yang, H.-D. Yeh, An analytical solution for describing the transient temperature distribution in an aquifer thermal energy storage system, Hydrol. Process. 24 (2010) $3676-3688$. 
[16] S.-Y. Yang, H.-D. Yeh, Solution for flow rates across the wellbore in a two-zone confined aquifer, J. Hydraul. Eng. 128 (2002) 175-183.

[17] G. S. Bödvarsson, C. F. Tsang, Injection and thermal breakthrough in fractured geothermal reservoirs, J. Geophys. Res. Solid Earth. 87 (1982) 1031-1048.

[18] B. Zerihun, K. Nils-Otto, K. Harald, K. Anne, Analytical and Numerical Solutions of Radially Symmetric Aquifer Thermal Energy Storage Problems, n. d.

[19] G. Evans, J. Blackledge, P. Yardley, Numerical methods for partial differential equations, Springer Science \& Business Media, 2012.

[20] Z. Li, Z. Qiao, T. Tang, Numerical Solutions of Partial Differential Equations-An Introduction to Finite Difference and Finite Element Methods, Cent. Res. Sci. Comput. Dep. Math. North Carolina. (2011).

[21] W. Zulehner, Lecture Notes for the Course Numerical Methods for Partial Differential Equations, (2006).

[22] D. N. Arnold, lecture notes on Numerical Analysis of Partial Differential Equations, (2005).
[23] Birhanu, Z. K., Kitterød, N.-O., Krogstad, H., Kværnø, A.: Analytical and Numerical Solutions of Radially Symmetric Aquifer Thermal Energy Storage Problems. Hydrol. Earth Syst. Sci. Discuss. 1-19 (2017).

[24] Ganguly, S., Mohan Kumar, M. S., Date, A., Akbarzadeh, A.: Numerical investigation of temperature distribution and thermal performance while charging-discharging thermal energy in aquifer. Appl. Therm. Eng. 115, 756-773 (2017).

[25] Gao, L., Zhao, J., An, Q., Wang, J., Liu, X.: A review on system performance studies of aquifer thermal energy storage. Energy Procedia. 142, 3537-3545 (2017).

[26] Ganguly, S., Kumar, M. S. M.: A numerical model for transient temperature distribution in an Aquifer Thermal Energy Storage system with multiple wells. Lowl. Technol. Int. 17, 179-188 (2015).

[27] Ganguly, S., Tan, L., Date, A., Kumar, M.: Numerical Investigation of Temperature Distribution in a Confined Heterogeneous Geothermal Reservoir Due to Injectionproduction. Energy Procedia. 110, 143-148 (2017). 